\title{
Community mental health teams for older people: variations in case mix and service receipt (1)
}

DOI:

10.1002/gps.4191

Document Version

Final published version

Link to publication record in Manchester Research Explorer

\section{Citation for published version (APA):}

Tucker, S., Wilberforce, M., Brand, C., Abendstern, M., Crook, A., Jasper, R., Stewart, K., \& Challis, D. (2015). Community mental health teams for older people: variations in case mix and service receipt (1). International journal of geriatric psychiatry, 30(6), 595-604. https://doi.org/10.1002/gps.4191

\section{Published in:}

International journal of geriatric psychiatry

\section{Citing this paper}

Please note that where the full-text provided on Manchester Research Explorer is the Author Accepted Manuscript or Proof version this may differ from the final Published version. If citing, it is advised that you check and use the publisher's definitive version.

\section{General rights}

Copyright and moral rights for the publications made accessible in the Research Explorer are retained by the authors and/or other copyright owners and it is a condition of accessing publications that users recognise and abide by the legal requirements associated with these rights.

\section{Takedown policy}

If you believe that this document breaches copyright please refer to the University of Manchester's Takedown Procedures [http://man.ac.uk/04Y6Bo] or contact uml.scholarlycommunications@manchester.ac.uk providing relevant details, so we can investigate your claim.

\section{OPEN ACCESS}




\title{
Community mental health teams for older people: variations in case mix and service receipt (I)
}

\author{
Sue Tucker, Mark Wilberforce, Christian Brand, Michele Abendstern, Anthony Crook, Rowan Jasper, \\ Karen Stewart and David Challis \\ Personal Social Services Research Unit, University of Manchester, Manchester, UK \\ Correspondence to: S. Tucker, E-mail: sue.tucker@manchester.ac.uk
}

\begin{abstract}
Objectives: The study sought to identify the characteristics of community-dwelling older people supported by community mental health teams (CMHTs) in England and, in particular, to determine whether there is a common threshold for CMHT entry and/or a core client group.

Methods: Data were collected about a random sample of 15 CMHTs' caseloads, including information about their sociodemographic characteristics, physical health, dependence, mental health, risks and service receipt. The sample was divided into 16 subgroups of people with similar needs for care (case types), and differences between teams were explored.

Results: Information was obtained for 1396 patients. Just under half had a functional mental health problem, slightly over a third an organic disorder, seven per cent both, and nine per cent no diagnosis. Considerable variation was found in teams' caseloads, and there was no evidence of a common caseload threshold. Two of the commonest case types represented people with functional diagnoses who were independent in activities of daily living (ADL) and had no/low levels of challenging behaviour. Another representing people with organic/mixed diagnoses, ADL dependence, challenging behaviour and at least one medium risk was also fairly common. The two case types that represented patients with the most complex needs accounted for more than a quarter of some teams' caseloads but less than a tenth of others.

Conclusions: It is wrong to assume that CMHTs all have similar caseloads. Commissioners must ensure that the network of services provided can meet the needs of all eligible patients, whilst more research is required on who such teams should target. Copyright (C) 2014 John Wiley \& Sons, Ltd.
\end{abstract}

Key words: community mental health teams; older people; caseloads; service provision History: Received 29 May 2014; Accepted 24 July 2014; Published online 9 September 2014 in Wiley Online Library (wileyonlinelibrary.com)

DOI: $10.1002 /$ gps.4191

\section{Introduction}

Recent decades have witnessed an increasing emphasis on the delivery of community-based mental health care. However, significant differences exist in the extent and configuration of provision (WHO/WPA, 1997; Jacob et al., 2007; Evans et al., 2012). The supply of community mental health services in England is amongst the most comprehensive in the world (Reifler and Cohen, 1998; Cahill et al., 2012). Indeed, right from their inception in the late 1940s, old-age psychiatry services in England aspired to a community orientation reflecting the postwar expansion of the mental hospital population, the increasing criticism of dehumanising long-stay institutions and the emergence of new psychotropic medications (Goffman, 1961; Dening, 1992; Hilton, 2005). Whilst many of the first consultants were based in hospitals, with beds in long-stay wards (Wattis et al., 1981), the subsequent pattern of service growth thus moved steadily towards the multidisciplinary assessment, treatment and support of individuals in the community (Von Abendorff et al., 1994), a shift reinforced by a variety of considerations, including costs and patients' preferences (DH, 2001; Knapp, 2005; Wanless et al., 2006).

In England today the core of specialist mental health provision for older people is formed by a network of community mental health teams (CMHTs) (Tucker et al., 2007a; Wilberforce et al., 2011) 
delivered by local mental health NHS Trusts (secondary care providers). Definitions of such teams vary, but typically make reference to their staff composition, function and target client group (Ovretveit, 1993; Onyett, 2003). The National Service Framework for Older People (NSFOP) and associated guidance, for example, recommended that CMHTs contain consultant psychiatrists, community mental health nurses, clinical psychologists, occupational therapists and social workers and concentrate on the diagnosis and treatment of patients with severe and/or complex needs, including people with depression and dementia. However, they also gave teams a number of other important roles, including the provision of outreach and advice to primary, residential, domiciliary, general hospital and day care services (DH, 2001; Lingard and Milne, 2004; DH and CSIP, 2005).

If the message here was that CMHTs should use their specialist skills with those that need them most, such guidance was not prescriptive, and whilst many CMHTs fulfil all these functions, in recent years, an increasing number of trusts have developed separate teams to undertake some of these activities. These include discrete memory assessment services, general hospital liaison and (less often) care home support teams (Lindesay et al., 2002; Thompsell, 2004; Holmes et al., 2010; Lawrence and Banerjee, 2010). As a result, CMHTs today are arguably less homogeneous than in previous years. To the best of our knowledge, however, no studies have evaluated the impact of such changes to CMHTs' casemix, whilst there are relatively few contemporary reports of who teams see (Abendstern et al., 2011). This is despite the fact that detailed knowledge of CMHTs' caseloads is essential for the identification of treatment needs and decisions about resource allocation and skill mix (Brown et al., 1996; Greenwood et al., 2000).

In the light of this omission, this paper, the first of two linked articles, aims to identify the characteristics of older people supported by CMHTs in England today, focusing on those patients living in their own homes. Key questions of interest include the extent to which there is a common threshold for CMHT entry and a core client group. Building on this, the second paper explores the pattern of services that this population receives, including whether people with different characteristics receive different inputs, and patients with similar profiles receive similar inputs from different teams. Whilst the examples all stem from England, the implications for workforce planning, service delivery and training will have a resonance for service providers and commissioners (funders) internationally.

\section{Method}

Information was collected about the caseloads of 15 CMHTs who participated in a research programme on the most appropriate and cost-effective ways of delivering care for older people with mental health problems (Challis et al., forthcoming). Nine teams (A-I) from across England participated in a study that investigated the factors that make for effective CMHT working, including integration and the role of the consultant (the CMHT study), whilst six teams (J-O) were involved in a study that explored the best combination of institutional and community services to provide in three areas of North-West England (the balance of care study). Although there were some small differences in the data collected, both sought to profile CMHT caseloads and took broadly the same approach.

A bespoke data collection tool was designed to ascertain information on a random sample of each team's caseload on a nominated day. This sought information about individuals' sociodemographic characteristics (age, gender, ethnicity and living situation), physical health, dependence, mental health (cognition, affect, behaviour and diagnosis) and level of risk and included a number of standardised measures: the modified Barthel Index (Mahoney and Barthel, 1965; Collin et al., 1988), the mini mental state examination (Folstein et al., 1975), the Minimum Data Set Cognitive Performance Scale (Morris et al., 1994), the Geriatric Depression Scale (Yesavage et al., 1983), the Hospital Anxiety and Depression Scale (Zigmond and Snaith, 1983); and the Patient Health Questionnaire-2 (Kreonke et al., 2003). Data were also collected about people's receipt of informal, specialist mental health and other community care. In Teams A-I, this information was sought for 120 individuals per team, whilst Teams J-O completed forms for all patients with organic or mixed organic and functional diagnoses, plus a one-in-four sample of people with solely functional mental health problems. Individuals under 65 were excluded, as were new patients who had not been fully assessed and long-term care home residents. Data collection took place between October 2010 and June 2011. Forms were completed by patients' key workers except in teams B and $\mathrm{H}$ (completed jointly by Clinical Studies Officers and key workers) and team F (completed by a Clinical Studies Officer alone).

Data from both studies were entered onto SPSS for Windows (version 19) and checked for errors. Subsequent analyses were conducted with Stata (versions 10 to 12 ). Where data permitted, critical gaps were filled by model-based and multiple imputation 
routines (refer to notes in Tables 2 and 3). Diagnoses were initially recorded as free text but later categorised into 10 broad groups plus a 'no formal diagnosis' category. Probability weights were applied to cases with functional diagnoses in teams $\mathrm{J}-\mathrm{O}$ to adjust for different sampling ratios.

The study samples were subsequently divided into relatively homogenous subgroups (case types) on the basis of four variables identified in the literature as likely to be important in determining the locus of and/or costs of their care (Tucker et al., 2013). Each of these had two levels, giving 16 possible combinations (Box 1).

Box 1. Attributes used to form the study case types

A dichotomous classification of diagnosis:

- Functional only;

- Organic or mixed organic and functional.

A two-level rating of dependence, based on a modified version of the Barthel Activities of Daily Living (ADL) Index:

- Independent (Barthel score 80-100);

- At least some degree of dependence (Barthel score 0-79).

A two-level classification of the extent to which people displayed seven behaviours known to be difficult for carers to manage (including agitation, aggression and resistance to care) based on a bespoke behaviour scale developed by the research team:

- None or low (score 0 or 1 );

- At least medium (score 2-14).

A two-level categorisation of the level of risk associated with the individual (including the risk of deliberate or accidental self-harm, self-neglect or abuse):

- No medium or high risks;

- At least one medium or high risk.

Differences between teams were explored using appropriate statistical tests. Analyses were design based, that is, accounted for the clustering of observations by teams (the primary sampling units) and the previously mentioned probability weights. However, given the purposeful selection and pooling of teams, no adjustments were made for different caseload sizes.
The intent was to investigate the existence of caseload variability at the national level, rather than to identify precise distributions.

Ethical approval for both studies was granted by Cambridgeshire 3 Research Ethics Committee (reference numbers $10 / \mathrm{H} 0306 / 43$ and $10 / \mathrm{H} 0306 / 51$ ) and research governance procedures in each participating organisation were fulfilled.

\section{Results}

Team profiles

The 15 CMHTs were located in 11 different mental health Trusts, including two formally integrated health and social care organisations. Teams exhibited considerable variation in both composition and context (Table 1). Most had between 10 and 20 members, although two ( $\mathrm{K}$ and $\mathrm{L}$ ) were noticeably larger. Team $\mathrm{K}$ included 15 (whole-time equivalent) support workers, whilst Team L had a particularly wide range of practitioners, including speech and language therapists, an assistant-grade psychologist and a physiotherapist. Nurses were the most numerous practitioner group in most teams. Social workers were present in 10 teams (the remainder were specifically selected for the CMHT study as not containing social care staff), whilst psychologists contributed one member per team at most.

\section{Caseload characteristics}

Information was collected about 1396 patients. Although the achieved sample sizes $(n=71-121)$ in Teams A-I often fell short of target, all bar two provided more than 100 cases. Tables 2 and 3 set out the full sample's diagnostic, sociodemographic, functional and clinical characteristics. Just under half had solely functional mental health problems (most commonly depression and/or anxiety) and just over a third organic disorders (most commonly dementia). As no definitive diagnosis was given for a large proportion of the latter (e.g. Alzheimer's disease or vascular dementia), these were included in the 'other dementia' category. A further seven per cent of patients had functional and organic disorders (hereafter described as 'mixed' diagnoses), whilst nine per cent had no recorded diagnosis.

Broad diagnostic profiles varied across teams. Whereas 72 per cent of Team C's sample had functional mental health problems, the same proportion of Team A's caseload had organic mental illness. There 


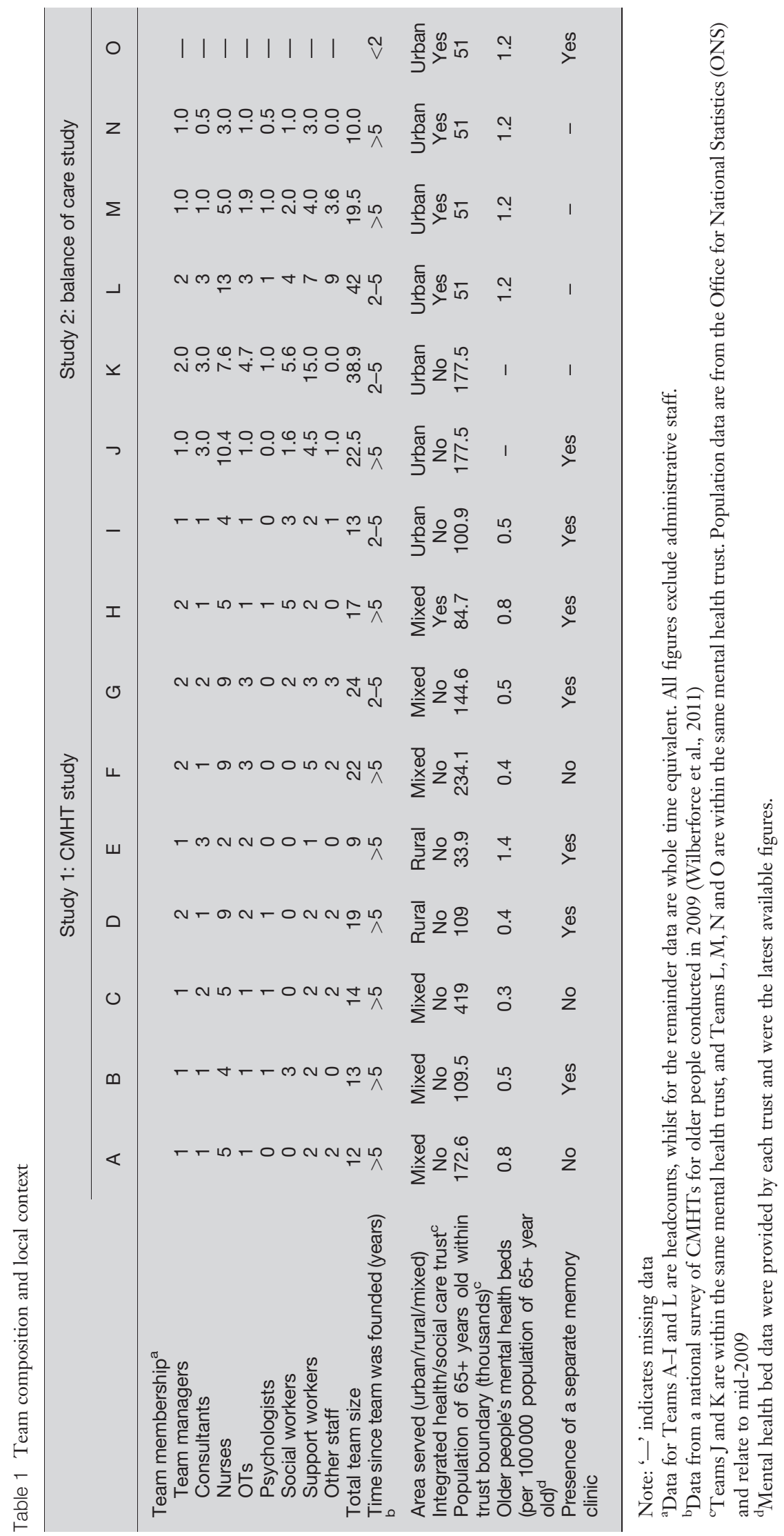


Table 2 CMHT caseloads: diagnostic and sociodemographic profiles ${ }^{\mathrm{a}}$

\begin{tabular}{|c|c|c|c|c|}
\hline & & Full sample \% & Min-Max \% across teams & Full sample $n$ \\
\hline \multirow[t]{12}{*}{ Psychiatric diagnosis } & Alzheimer's disease & 10.4 & $1.1-42.3$ & 179 \\
\hline & Vascular dementia & 8.6 & $1.0-18.8$ & 143 \\
\hline & Other dementia & 11.0 & $1.7-33.0$ & 186 \\
\hline & Other/Multiple organic illness & 4.5 & $0.0-15.6$ & 75 \\
\hline & Total with organic illness & 34.5 & $16.8-71.9$ & 583 \\
\hline & Depression/Anxiety/Related disorders & 29.6 & $5.3-55.8$ & 359 \\
\hline & Other affective disorders & 6.7 & $0.0-21.3$ & 72 \\
\hline & Schizophrenia & 5.9 & $1.4-21.1$ & 67 \\
\hline & Other/Multiple functional illness & 7.1 & $1.0-21.5$ & 72 \\
\hline & Total with functional illness & 49.3 & $15.4-72.1$ & 570 \\
\hline & Mixed organic/Functional illness & 7.1 & $0.9-12.7$ & 108 \\
\hline & No formal diagnosis in place & 9.1 & $0.0-26.4$ & 135 \\
\hline \multirow[t]{2}{*}{ Gender } & Female & 64.8 & $54.6-73.7$ & 903 \\
\hline & Male & 35.2 & $26.3-45.4$ & 489 \\
\hline \multirow[t]{3}{*}{ Age } & $65-74$ & 32.8 & $18.1-44.7$ & 422 \\
\hline & $75-84$ & 46.5 & $30.6-57.0$ & 643 \\
\hline & $85+$ & 20.7 & $8.9-38.8$ & 320 \\
\hline \multirow[t]{2}{*}{ Ethnicity } & White British & 94.9 & $75.1-100$ & 1310 \\
\hline & Black and minority ethnic groups & 5.1 & $0.0-24.9$ & 72 \\
\hline \multirow{3}{*}{$\begin{array}{l}\text { Usual place of } \\
\text { residence }\end{array}$} & Own home: lives alone & 46.2 & $26.2-61.1$ & 615 \\
\hline & Own home: lives with others & 47.1 & $33.9-72.3$ & 572 \\
\hline & Other including extra care housing & 6.6 & $0.4-20.4$ & 95 \\
\hline Informal care hours & None & 38.0 & $21.4-61.1$ & 456 \\
\hline \multirow[t]{3}{*}{ per week } & $1-7$ & 26.1 & $12.2-40.4$ & 335 \\
\hline & $8-20$ & 16.0 & $8.3-27.0$ & 241 \\
\hline & $21+$ & 19.9 & $9.5-31.7$ & 298 \\
\hline \multirow[t]{2}{*}{ Previous occupation ${ }^{\mathrm{b}}$} & $\begin{array}{l}\text { Managerial, professional and technical } \\
\text { occupations }\end{array}$ & 29.8 & $0.0-51.0$ & 250 \\
\hline & Other & 70.2 & $0.0-41.5$ & 615 \\
\hline
\end{tabular}

${ }^{a}$ Weighted percentages to adjust for different sampling ratios applied to functional and organic cases, as described in the main text.

boccupations coded according to ONS Standard Occupational Classifications. 'Other' incorporates administrative, skilled trades, personal service, sales, machine operatives, and elementary occupations.

Table 3 CMHT caseloads: functional and clinical profiles ${ }^{\mathrm{a}}$

\begin{tabular}{|c|c|c|c|c|}
\hline & & Full sample \% & Min-Max \% across teams & Full sample $n$ \\
\hline \multirow{4}{*}{ Activities of daily living ${ }^{\text {b }}$} & Independent & 74.1 & $59.6-91.3$ & 994 \\
\hline & Minimal help needed & 15.0 & $5.8-27.0$ & 223 \\
\hline & Partially dependent & 7.0 & $2.6-16.0$ & 102 \\
\hline & Totally/Very dependent & 4.0 & $0.0-14.8$ & 61 \\
\hline \multirow[t]{3}{*}{ Cognition $^{c}$} & Intact/Only mild impairment & 69.1 & $46.3-83.4$ & 866 \\
\hline & Moderate impairment & 23.1 & $11.0-42.8$ & 370 \\
\hline & Severe impairment & 7.8 & $1.6-19.0$ & 123 \\
\hline \multirow[t]{3}{*}{ Challenging behaviour ${ }^{\mathrm{b}}$} & Low & 44.4 & $17.7-72.1$ & 594 \\
\hline & Medium & 51.1 & $26.0-77.0$ & 719 \\
\hline & High & 4.6 & $1.6-12.8$ & 72 \\
\hline \multirow[t]{2}{*}{ Risks } & At least one high risk & 13.2 & $4.8-29.9$ & 179 \\
\hline & No high risks & 86.8 & $70.7-95.2$ & 1180 \\
\hline
\end{tabular}

${ }^{a}$ Weighted percentages to adjust for different sampling ratios applied to functional and organic cases.

${ }^{b}$ Includes small fractions of imputed values (single model-based imputation to fill gaps caused by a listwise deletion problem when constructing summary measures, drawing on reported question battery items); approximate fraction missing: ADL (11\%), behaviour score $(6 \%)$

${ }^{\mathrm{c}}$ Estimated cognition rating is based on 20 multiple imputations (truncated regression) of mini mental state examination (MMSE) scores ( $<12$ months old) and a fraction of staff-rated cognition estimates (in teams A-J); approximate fraction missing was $48 \%$. In teams $\mathrm{K}$ to $\mathrm{O}$, the rating is derived from the Minimum Data Set cognitive performance score. It is likely that the 'moderate impairment' category has been slightly overestimated because of limitations in predicting MMSE scores, whilst the 'severe impairment' category is believed to be a reliable estimate. Multiple imputation confidence: $65-73 \%, 20-27 \%, 6-9 \%$ 
were also marked differences in the percentage with specific diagnoses, such as schizophrenia (1\% in Team $\mathrm{O}$ and $21 \%$ in Team $\mathrm{G}$ ), and no recorded diagnosis (zero in Team I and 26\% in Team H).

The mean (standard deviation) age of the full sample was 78 years (7.1), with approximately a fifth $85+$ years. As expected, age varied with diagnosis. Just 13 per cent of patients with functional mental health problems were $85+$ years, compared with 28 per cent with organic or mixed diagnoses (design-based $\chi^{2}, F$ $(1.25,17.50)=28.07, p<0.001)$. In contrast, 44 per cent of individuals with functional disorders were under 75 years, compared with 22 per cent with other diagnoses. Five per cent of the full sample were from minority ethnic groups (ranging from none in Team E to $25 \%$ in Team N), and proportionately more patients from minority ethnic groups than white British patients had functional mental illnesses (68 vs 53\%; design-based $\left.\chi^{2}, F(1,14)=6.51, p=0.023\right)$.

A similar proportion of the sample lived alone as lived with others. However, patients with functional mental health problems were significantly more likely to live alone than those with other disorders (55 vs $39 \%$; design-based $\chi^{2}, F(1,14)=22.53$, $p<0.001)$ and, correspondingly, less likely to receive informal care (44 vs $81 \%$; design-based $\chi^{2}$, $F(1,14)=101.18, p<0.001)$. Just seven per cent of the sample lived in extra care housing/supported accommodation, varying from a nominal proportion in Team K to 20 per cent in Team I, dependent on local availability.

Finally, although three quarters of the sample were independent in ADLs, there was significant variation across teams and a strong association with diagnosis. As expected, patients with organic or mixed diagnoses were significantly less likely to be ADL independent than those with functional mental health problems (60 vs $86 \%$; design-based $\chi^{2}, F(1$, $14)=28.86, p<0.001)$. Challenging behaviour and risk were similarly associated with diagnosis. For example, 17 per cent of individuals with organic or mixed diagnoses presented high levels of challenging behaviour, compared with eight per cent with functional diagnoses (design-based $\chi^{2}, \quad F(1$, 14) $=10.34, p=0.006)$.

\section{Case types}

Of the 16 case types used to categorise people with similar care needs, all were populated. As anticipated, however, some combinations of characteristics were more common than others. Table 4 outlines the

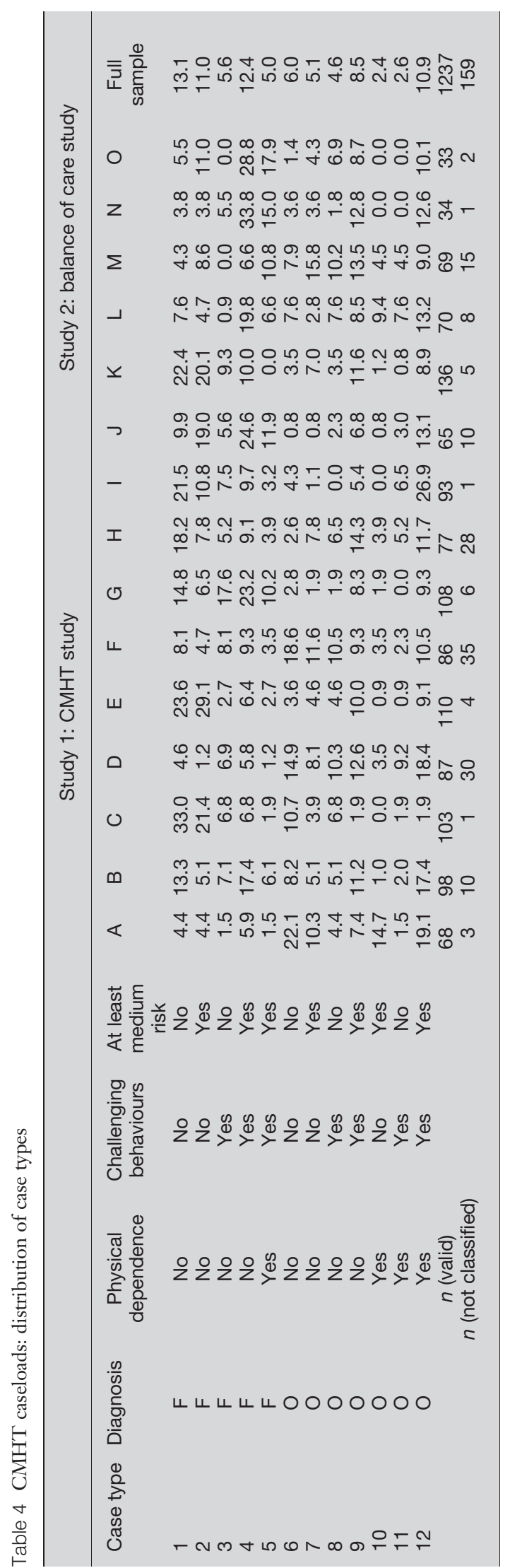


distribution of the 12 most prevalent groups, which between them accounted for nearly 90 per cent of the total sample. No excluded case type captured more than five per cent of any team's sample.

The prevalence of different case types varied considerably across teams, and no single case type dominated the full sample. Two of the largest case types (1 and 2), representing patients with functional diagnoses who were ADL independent and presented no/low levels of challenging behaviour (albeit case type 2 had at least one medium risk), together accounted for just under a quarter of the full sample. This included the majority of Team C and E's caseloads but less than a tenth of Teams A, D and N's. Case type 4 (functional diagnosis, not ADL dependent but challenging behaviour and risks) was also relatively common, particularly in Teams J, $\mathrm{N}$ and $\mathrm{O}$.

Of case types representing people with organic or mixed diagnoses, case type 12 (ADL dependent, challenging behaviour and one or more medium risk) was most prevalent. However, whilst this captured over a quarter of Team I's caseload, it made up just two per cent of Team C's. Similar breadth of prevalence was found at the other end of the needs spectrum. Thus, case type 6 (no ADL dependence, challenging behaviour or medium/high risks) represented approximately a fifth of the samples from Teams $\mathrm{A}$ and $\mathrm{F}$ but only a nominal proportion from Teams $\mathrm{J}$ and $\mathrm{O}$. Indeed, considerable variation was seen in the overall proportion of people with low and high-level needs on different teams' caseloads. Thus, those two case types that theoretically represented patients with the lowest level needs (types 1 and 6) captured more than a quarter of Team A, E, F, I and K's samples and more than four-tenths of Team C's but less than a tenth of Team $\mathrm{N}$ and O's. Correspondingly, those two case types with the most complex needs (types 5 and 12) accounted for more than a quarter of the caseload in Teams I, J, N and $\mathrm{O}$ but less than a tenth in Teams $\mathrm{C}$ and $\mathrm{K}$. No single case type dominated the profiles of Teams B and M. The number of small cells in Table 4 precluded a complete statistical appreciation of the variability in this data. However, tests of association between more numerous case types and teams highlighted its non-random nature. For example, a logistic regression predicting membership of case type 12 suggested that Team I was three times more likely than the average team to see such individuals (design-based OR 3.27, $F(1,14)=130.54, t=11.43, p<0.001)$.

\section{Discussion}

This paper provides a unique account of older people supported by CMHTs in England at a time when mental health services were changing rapidly and acts as a benchmark against which provision at other time points and in other countries can be compared. Moreover, whereas most past descriptions of CMHTs' caseloads in working age and older people's services have focused on diagnosis, the case-type methodology employed here sought to move beyond this to identify groups of people with similar needs for care (Brown et al., 1996; Greenwood et al., 2000; Hunter et al., 2002). The picture that it presents is mixed. The fact that some case types were more prevalent than others points to the existence of certain key groups. However, no clear evidence was found of a common caseload threshold, and differences between teams were more marked than similarities.

\section{Methodological considerations}

The teams described in this paper were purposively selected for their respective studies and were not necessarily representative of England as a whole. Furthermore, as not all teams saw younger adults with dementia or care home residents, the analysis related only to community-dwelling patients aged $65+$ years. Nevertheless, in terms of size, the teams represented the full range of CMHTs responding to a large national survey (Wilberforce et al., 2011) and accepted referrals with a wide range of mental health problems, whilst there was no indication that differences in caseloads were attributable to the sociodemographic or economic characteristics of their catchment areas.

The use of data from two separate studies necessitated a certain amount of recoding. However, whereas most past caseload reports are limited to a single team or organisation (Wooff et al., 1986; Brown et al., 1996; Hunter et al., 2002), this analysis drew on information from multiple organisations. The high proportion of data collected by professionals that one might expect to be well informed about patients' current circumstances is a further strength, and whilst few checks were made on the accuracy of the data, there is no reason to believe any inter-rater differences varied systematically from team to team.

Although, ideally, it would have been desirable to further refine the case types by including a rating of symptom severity, gaps in the availability of measures of affect and cognition precluded this. There was 
also variability in the quality of local record-keeping systems, whilst the distribution of cases with no primary diagnosis was not randomly spread: patients with missing diagnosis were more likely to be relatively new and/or complex cases. However, no association was found between teams with less complex cases and teams with higher levels of missing diagnoses.

\section{Implications for practice and commissioning}

Although CMHTs are commonly agreed to form the heart of community mental health services for older people in England, this paper suggests that it would be wrong to assume that they all have similar caseloads. Whereas some teams focused on people with multiple, complex needs, others saw a much wider range of individuals. Similarly, some teams supported a majority of people with dementia, whilst others saw relatively few.

Such divergence may, in part, be because of differences in the broader range of services available in different localities and is not necessarily of concern (Banerjee and Chan, 2008). In some (but not all) areas, for example, the existence of discrete memory services may at least partly explain the relative lack of patients with dementia with lower level needs, whilst the presence of comprehensive primary mental health services might account for a comparative lack of people with less complex depression in others. Nevertheless, the extent of variation in caseload profiles tends to suggest that this is not the whole story and that such variation is more likely to reflect a lack of strong evidence to inform commissioning (McCrae and Banerjee, 2011). Indeed, given the direct costs of CMHTs for older adults in England amount to $\mathfrak{E} 227 \mathrm{~m}$ per annum (Naylor and Bell, 2010), the findings raise a number of questions about who such teams should target and who is best served by other, allied, services. Whilst research suggests that primary and specialist mental health staff generally agree who should take responsibility for patients with different needs (Tucker et al., 2007b), only a handful of controlled studies have measured CMHTs' clinical effectiveness with particular client groups (e.g. Jenkins and Macdonald, 1994; Hinchliffe et al., 1995; Banerjee et al., 1996), and there are still fewer studies of comparative service provision (Woods et al. 2003; McCrae and Banerjee, 2011).

If the desire to make the best use of resources is one argument for delineating CMHTs' caseloads, another is the need to ensure that the roles and responsibilities of different service elements are clear to referrers (Naylor and Bell, 2010), for where this is not the case, the potential for people to slip between services will be enhanced. Similarly, commissioners must be assured that the network of services provided can meet the needs of the whole client group. With this in mind, it is interesting to note that in comparison with most previous studies, our findings suggest that there has been a decline in the proportion of people with organic disorders on CMHTs' caseloads over time (Junaid and Bruce, 1994; Abendstern et al., 2006), perhaps because, as indicated previously, more specialist services now meet their needs.

Finally, in the light of arguments that the provision of specialist services for older adults sits uncomfortably with current age discrimination legislation and that this group is being denied access to a range of services widely available to younger adults (including assertive outreach, intensive home treatment and psychological therapies), it is interesting to consider how the development of 'age-inclusive' services might impact on the mix of older people seen by CMHTs (Anderson et al., 2009; Royal College of Psychiatrists, 2009a, 2009b). Indeed, since these data were collected, at least two of the teams have been absorbed into single all-adult teams. One obvious concern is that when considered alongside the needs of younger people, older people might be overlooked, for in contrast to the NSFOP, the National Service Framework for Mental Health was highly prescriptive in prioritising people with serious and enduring mental illness, particularly schizophrenia (DH, 1999). The profile of disorder in older people is, however, qualitatively and quantitatively different from that in younger adults, whose needs are less likely to be complicated by the presence of physical illness and disability (Banerjee and Chan, 2008; Anderson et al., 2009). Referral criteria that work for younger adults will not necessarily work for older ones. Indeed, it should not be forgotten that a further driver of the emergence of old-age psychiatry as a specialist discipline was the need to provide for those older people eschewed by general psychiatry. As a report on age discrimination in mental health points out, 'equity is not treating all people the same'. Rather, 'equity recognises that different needs should be addressed differently but fairly' (Anderson et al., 2009 p. 13).

\section{Conclusion}

This paper has suggested that CMHTs' caseloads vary by team, have changed over time and are likely to 
change further in the future. It has also considered a number of possible explanations for this and explored some of the potential implications for policy makers and commissioners. In the second of these linked papers, we next consider the immediate consequences for patients themselves in terms of the support that they receive and the lessons for service providers regarding teams' staffing, roles and training.

\section{Conflicts of interest}

None declared.

\section{Key points}

- Informed decisions about resource allocation and skill mix require detailed knowledge of CMHT caseloads. However, there are few contemporary reports of who such teams see.

- This study found large differences in the sociodemographic, dependence and diagnostic profiles of CMHTs' community-dwelling caseloads.

- Patients with an organic diagnosis constituted more than two-thirds of some teams' caseloads but less than a fifth of others. The proportion categorised with the most complex needs also varied greatly.

- It is wrong to assume that CMHTs all have similar caseloads. Commissioners must ensure that the network of services provided can meet the needs of all eligible patients, whilst more research is needed on who such teams should target.

\section{Ethics statement}

Ethical approval for the two studies from which this data were drawn was granted by Cambridgeshire 3 Research Ethics Committee (reference numbers $10 / \mathrm{H} 0306 / 43$ and 10/H0306/51) and research governance procedures in each participating organisation were fulfilled.

\section{Acknowledgements}

We are very grateful to the CMHT staff who participated in this research. This paper presents independent research funded by the National Institute for Health Research (NIHR) under its Programme Grants for Applied Research scheme (RP-PG-0606-1109). The views expressed in this publication are those of the authors and not necessarily those of the NHS, the NIHR or the Department of Health.

\section{References}

Abendstern M, Reilly S, Hughes J, et al. 2006. Levels of integration and specialisation within professional community teams for people with dementia. Int J Geriatr Psychiatry 21(1): 77-85.

Abendstern M, Harrington V, Brand C, et al. 2011. Community Mental Health Teams for Older People: Structures, Processes and Outcomes. A systematic literature review. Filed as Discussion Paper M243. Personal Social Services Research Unit, The University of Manchester: Manchester.

Anderson D, Banerjee S, Barker A, et al. 2009. The need to tackle age discrimination in mental health. A compendium of evidence. Royal College of Psychiatrists: London.

Banerjee S, Chan J. 2008. Organization of old age psychiatric services. Psychiatry 7(2): 49-54.

Banerjee S, Shamash K, Macdonald AJD, et al. 1996. Randomised controlled trial of effect of intervention by psychogeriatric team on depression in frail elderly people at home. BMJ 313(7064): 1058-1061.

Brown P, Challis D, Von Abendorff R. 1996. The work of a community mental health team for the elderly: Referrals, caseloads, contact history and outcomes. Int $J$ Geriatr Psychiatry 11(1): 29-39.

Cahill S, O'Shea E, Pierce M. 2012. Creating Excellence in Dementia Care. A research review for Ireland's National Dementia Strategy. Trinity College School of Social Work and Social Policy and National University of Ireland: Dublin and Galway.

Challis D, Tucker S, Wilberforce M, et al. Forthcoming. National trends and local delivery in old age mental health services: towards an evidence base. A mixed methodology study of the balance of care approach, community mental health teams and specialist mental health outreach to care homes. Programme Grants Appl Res.

Collin C, Wade DT, Davies S, et al. 1988. The Barthel ADL Index: A reliability study. Int Disabil Stud 10(2): 61-63.

Dening T. 1992. Community psychiatry of old age: A UK perspective. Int J Geriatr Psychiatry 7(10): 757-766.

Department of Health. 1999. National Service Framework for Mental Health. Modern Standards and Service Models. Department of Health: London.

Department of Health. 2001. National Service Framework for Older People. Department of Health: London.

Department of Health, Care Services Improvement Partnership. 2005. Everybody's business. Integrated mental health services for older adults. A service development guide. Department of Health: London.

Evans S, Huxley P, Baker C, et al. 2012. The social care component of multidisciplinary mental health teams: a review and national survey. J Health Serv Res Policy 17(Suppl 2): 23-29.

Folstein MF, Folstein SE, McHugh PR. 1975. "Mini-mental state". A practical method for grading the cognitive state of patients for the clinician. J Psychiatr Res 12(3): 189-198.

Goffman E. 1961. Asylums: Essays on the social situation of mental patients and other inmates. Doubleday: New York.

Greenwood N, Chisholm B, Burns T, et al. 2000. Community mental health team case-loads and diagnostic case-mix. Psychiatr Bull 24(8): 290-293.

Hilton C. 2005. The Origins of Old Age Psychiatry in Britain in the 1940s. Hist Psychiatry 16(63 pt 3): 267-289.

Hinchliffe AC, Hyman IL, Blizard B, et al. 1995. Behavioural complications of dementia - can they be treated? Int J Geriatr Psychiatr 10(10): 839-847.

Holmes J, Montaňa C, Powell G, et al. 2010. Liaison mental health services for older people: A literature review, service mapping and in-depth evaluation of service models. HMSO: London.

Hunter MD, Jadresic D, The Audit Working Group. 2002. Two weeks in the life of a community mental health team: a survey of case-mix and clinical activity in the north-west of Sheffield. Psychiatr Bull 26(1): 9-11.

Jacob KS, Sharan P, Mirza I, et al. 2007. Mental health systems in countries: where are we now? Lancet 370(9592): 1061-1077.

Jenkins D, Macdonald A. 1994. Should general practitioners refer more of their elderly depressed patients to psychiatric services? Int J Geriatr Psychiatr 9(6): 461-465.

Junaid O, Bruce J. 1994. Providing a community psychogeriatric service - models of community psychiatric nursing provision in a single health district. Int J Geriatr Psychiatr 9(9): 715-720.

Knapp M. 2005. Money talks: Nine things to remember about mental health financing. J Ment Health 14(2): 89-93.

Kroenke K, Spitzer RL, Williams J. 2003. The Patient Health Questionnaire-2: Validity of a two-item depression screener. Med Care 41(11): 1284-1292. 
Lawrence V, Banerjee S. 2010. Improving care in care homes: A qualitative evaluation of the Croydon care home support team. Aging Ment Health 14(4): 416-424.

Lindesay J, Marudkar M, van Diepen E, et al. 2002. The second Leicester survey of memory clinics in the British Isles. Int J Geriatr Psychiatr 17(1): 41-47.

Lingard J, Milne A. 2004. Integrating Older People's Mental Health Services: Community Mental Health Teams for Older People. A Commentary and Resource Document. Department of Health: London.

Mahoney FI, Barthel DW. 1965. Functional evaluation: the Barthel Index: a simple in dex of independence useful in scoring improvement in the rehabilitation of the chronically ill. Md State Med J 4: 61-65.

McCrae N, Banerjee S. 2011. The challenge of evaluating mental health services for older people. Int J Geriatr Psychiatr 26(6): 551-557.

Morris JN, Fries BE, Mehr DR, et al. 1994. Cognitive Performance Scale. J Gerontol 49(4): M174-182.

Naylor C, Bell A. 2010. Mental health and the productivity challenge: Improving quality and value for money. King's Fund: London.

Onyett S. 2003. Teamworking in Mental Health. Palgrave Macmillan: Basingstoke.

Ovretveit J. 1993. Coordinating Community Care: Multidisciplinary Teams and Care Management. Open University Press: Buckingham.

Reifler BV, Cohen W. 1998. Practice of geriatric psychiatry and mental health services for the elderly: Results of an international survey. Int Psychogeriatr 10(4): 351-357.

Royal College of Psychiatrists. 2009a. Age discrimination in mental health services: Making equality a reality. Royal College of Psychiatrists' position statement PS2/2009. Royal College of Psychiatrists: London.

Royal College of Psychiatrists. 2009b. The need to tackle age discrimination in mental health: A compendium of evidence. Royal College of Psychiatrists: London.

Thompsell A. 2004. The care home flying squad. J Dement Care 12(5): 12-13.
Tucker S, Baldwin R, Hughes J, et al. 2007a. Old age mental health services in England: Implementing the National Service Framework for Older People. Int J Geriatr Psychiatr 22(3): 211-217.

Tucker S, Hughes J, Scott J, et al. 2007b. Commissioning services for older people with mental health problems: Is there a shared vision? J Integr Care 15(2): 3-12.

Tucker S, Brand C, Wilberforce M, et al. 2013. The balance of care approach to health and social care planning: Lessons from a systematic literature review. Health Serv Manage Res 26(1): 18-28.

Von Abendorff R, Challis D, Netten A. 1994. Staff activity patterns in a community mental health team for older people. Int J Geriatr Psychiatr 9(11): 897-906.

Wanless D, Forder J, Fernandez JL, et al. 2006. Securing Good Care for Older People: Taking a Long-Term View. King's Fund: London.

Wattis J, Wattis L, Arie T. 1981. Psychogeriatrics: A national survey of a new branch of psychiatry. BMJ 282(6275): 1529-1533.

Wilberforce M, Harrington V, Brand C, et al. 2011. Towards integrated community mental health teams for older people in England: Progress and new insights. Int J Geriatr Psychiatr 26(3): 221-228.

Woods RT, Wills W, Higginson IJ, et al. 2003. Support in the community for people with dementia and their carers: A comparative outcome study of specialist mental health service interventions. Int J Geriatr Psychiatr 18(4): 298-307.

Wooff K, Goldberg DP, Fryers T. 1986. Patients in receipt of community psychiatric nursing care in Salford 1976-82. Psychol Med 16(2): 407-414.

World Health Organisation, World Psychiatric Association. 1997. Organisation of care in psychiatry of the elderly: A technical consensus statement. WHO and WPA: Geneva.

Yesavage JA, Brink TL, Rose TL, et al. 1983. Development and validation of a geriatric depression screening scale: A preliminary report. J Psychiatr Res 17(1): 37-49.

Zigmond AS, Snaith RP. 1983. The Hospital Anxiety and Depression Scale. Acta Psychiatr Scand 67(6): 361-370. 